\title{
Migration contemporaine, politique locale et construction de l'autochtonie au Cameroun : le cas des Pouakam du royaume bamoun
}

\author{
By Ibrahim Mouiche*
}

\begin{abstract}
The Pouakam constitute a small minority ethnic group arrived and established after the independence of Cameroon intervened in 1960, in a continuous territorial enclave included between the Bamun and the Banso kingdoms. In spite of the fact that this migration is contemporary, they have acquired here the status of autochthons. This privileged position of the Pouakam seems to contradict the classical migrationist and autochthonist theories, especially since other immigrant groups who arrived well before them are still considered as non-natives. If this position leans on the theory of "mobile continuity" and of "désert humain," it differentiates itself by the weight it grants to the interactions of ethnic groups unlike the classical theory widely based on the colonial determinism. The study is essentially about the Pouakam of the Bamoun kingdom.
\end{abstract}

Résumé : Les Pouakam constituent un groupe ethnique très minoritaire, arrivés et établis définitivement après l'indépendance du Cameroun intervenue en 1960, dans une enclave territoriale continue et comprise entre les royaumes bamoun et banso. Malgré cette migration contemporaine, ils ont acquis ici le statut d'autochtones. Cette position privilégiée des Pouakam semble contrarier les thèses classiques migrationnistes et de l'autochtonie, ce d'autant plus que d'autres groupes immigrants arrivés bien avant eux sont toujours considérés ici comme des allogènes. Si elle s'appuie sur la thèse de " continuité mobile » et du "désert humain », elle s'en démarque par le poids qu'elle accorde à l'interaction des groupes ethniques au contraire de la thèse classique largement assise sur le déterminisme colonial. L'étude porte essentiellement sur les Pouakam du royaume bamoun.

$* * *$

* Ibrahim Mouiche : enseignant-chercheur et professeur de science politique à l'Université de Yaoundé II au Cameroun. Actuellement lauréat de la Fondation Alexander von Humboldt et chercheur-résident de GIGA Institute of African Affairs à Hambourg, j'ai profité de ce séjour scientifique pour écrire et finaliser cet article. Je saisis cette occasion pour exprimer toute ma gratitude à la Fondation Humboldt et à GIGA pour leur soutien financier et logistique. Email : ibrahimi99@yahoo.com. 


\section{Introduction}

Les Pouakam sont ces populations qu'on retrouve dans le rebord occidental des piedmonts du massif Mbam dans une dépression continue comprise entre à l'est, l'arrondissement de Bangourain dans le département du Noun, et à l'ouest, l'arrondissement de Jakiri, département $\mathrm{du}$ Bui ${ }^{1}$. Ils constituent un groupe ethnique très minoritaire, arrivé et établi définitivement vers la fin de la décennie 1960 en pays bamoun et banso ainsi que l'atteste leur procès migratoire. Malgré cette migration après l'indépendance du Cameroun intervenue en 1960, ils ont acquis ici le statut des «gens du pays », expression empruntée à Michel Bozon ${ }^{2}$ pour désigner les autochtones. Cette position privilégiée des Pouakam a aiguisé notre curiosité scientifique parce qu'elle semble contrarier les thèses classiques migrationnistes et de l'autochtonie largement assisses sur la continuité historique d'un groupe dans une région avant la colonisation, ce d'autant plus que d'autres groupes immigrants arrivés bien avant eux sont toujours considérés ici comme des allogènes ainsi que nous le verrons. Notre interrogation est d'autant plus pertinente que depuis les années 1990, la recherche sur les politiques de l'ethnicité, de l'appartenance et de la citoyenneté en Afrique s'est élargie à cette problématisation de «l'autochtonie». En employant cette catégorie, on a pu mettre en évidence et désigner certaines homologies observables dans les conflits qu'ont connus des pays comme la Côte d'Ivoire, où l'on s'est battu au nom de l'ivoirité, l'Afrique du Sud, où ont été agressés des travailleurs étrangers, ou encore le Rwanda, où les Tutsi ont été présentés comme des allochtones « hamites » d'origine « éthiopique »; la notion d'autochtonie a également été employée pour décrire les tensions observées au Cameroun, où les droits civils et politiques des «allochtones » ont été remis en question ${ }^{3}$.

C'est à une problématique non conflictogène ou de crises que s'inscrit cette étude sur l'autochtonie des Pouakam, ce peuple appartenant à la même aire culturelle semi-bantoue ${ }^{4}$

1 Le département du Noun est situé dans la région francophone de l'Ouest du Cameroun; il épouse les mêmes frontières historiques que le royaume bamoun. Le département du Bui, quant à lui, fait partie de la région anglophone du Nord-Ouest et à l'instar du Noun avec le royaume bamoun, il garde les mêmes frontières historiques que le royaume banso.

2 Bozon, Michel, « Chasse, territoire, groupements de chasseurs » in Études rurales, no. 87/88, Jul. Dec., 1982, pp.335-342.

3 Cutolo, Armando, « Populations, citoyennetés et territoires. Autochtonie et gouvernementalité en Afrique » in Politique africaine $\mathrm{n}^{\circ} 112$, décembre 2008, p. 6.

4 Les semi-bantous appartiennent à un même cercle culturel. A l'intérieur de ce cercle, une même civilisation conserve partout ses traits essentiels, avec des nuances multiples imposées par la physionomie des groupes : organisation politique très hiérarchisée couronnée par un chef puissant, assisté d'un conseil de notables; regroupement des habitants en multiples sociétés. Les variations les plus significatives sont apportées par l'étendue spatiale de ces unités. Le dynamisme politique, militaire ou démographique différent a conduit soit à la constitution d'ensembles importants, c'est le cas des royaumes bamoun, nso ou bali, soit à un morcellement en chefferies de tailles très diverses dont certaines étaient en position dominante par rapport à leurs voisines alors que les autres ne pouvaient que se défendre, avec un succès inégal, contre les prétentions des premières. C'était le cas général du pays bamiléké et de la plupart des peuples de l'actuelle région du Nord-Ouest. Champaud, Jacques, Villes et campagnes du Cameroun, Paris, 1983, p. 35-36. 
avec les Bamoun, les Bamiléké, les Banso, les Tikar, etc., bien que méconnu des travaux des anthropologues et autres historiens. Des entretiens que nous avons eus dans cette localité, cette communauté s'appelle Pouakam ou Mbokam selon qu'on est en territoire bamoun ou en territoire banso et respectivement par les populations bamoun et celles banso. De leur côté, les concernés préfèrent l'ethnonyme Pouakam et considèrent Mbokam comme un sobriquet à eux accolé par les Banso avec lesquels ils cohabitent. Les Pouakam sont cependant divisés en deux tribus avec chacune un chef bien que fortement apparentées: d'un côté les Nchingong circonscrits uniquement en territoire bamoun; de l'autre, les Nzerem, qu'on retrouve de part et d'autre en territoire bamoun (attenant les Nchingong) et en territoire banso. L'on note toutefois une continuité territoriale entre les deux groupes, séparés seulement par une rivière. Côté bamoun, les deux peuples ont formé chacun, un village : Koupouakam I pour les Nchingong et Koupouakam II pour les Nzerem. Mais, c'est en territoire banso à Fonkigham que se trouve le palais du chef des Nzerem. Les Nzerem sont donc établis dans deux quartiers : Fonkigham en territoire banso et Koupouakam II (ou Mangori selon les Banso) en territoire bamoun. Résidant en territoire banso, le chef des Nzerem reste sous la tutelle du roi (fon) des Banso alors que ses populations de Koupouakam II sont soumises coutumièrement plutôt au sultanroi des Bamoun, parallèlement avec les Nchingong et leur chef. Au plan linguistique, les deux tribus partagent une même langue avec cependant une petite variation dialectale. Cette langue est très proche du parler bamoun ${ }^{5}$. Si à Fonkigham, les Pouakam cohabitent avec une forte colonie agricole banso, en pays bamoun, à Koupouakam I et II, ils restent isolés, bien loin des premiers villages bamoun que surplombe le massif $\mathrm{Mbam}^{6}$.

5 Elle s'inscrit ainsi dans l'ensemble linguistique bandam-nkom et mbam-mkam qu'on retrouve également chez les Bafanji, Bapessi, Bamali, Bambalang, Bangolan de la plaine de Ndop dans le département voisin du Ngo Ketunja (région du Nord-Ouest).

6 Les Pouakam connaissent une très forte marginalisation; celle-ci est liée à leur enclavement et leur position excentrée par rapport aux grands axes routiers et centres administratifs que sont Bangourain et Jakiri. Au sein de l'arrondissement de Bangourain, les villages Koupouakam I et II sont enclavés. Pour rallier ces deux villages, l'on doit transiter par Jakiri dans la région du Nord-Ouest avec des effets induits sur le coût du transport puisqu'il faut faire deux jonctions : le tronçon Pouakam-Jakiri puis Jakiri-Bangourain. Certes, sur le plan administratif, en cas de survenance d'un problème nécessitant l'intervention de l'administration, c'est à Bangourain que les Pouakam du pays bamoun se dirigent pour en chercher solution et ceux de Fonkigham (territoire banso) à Jakiri; mais quotidiennement, lorsqu'il s'agit des actes personnels tels que l'établissement de la carte nationale d'identité, d'actes d'état civil, tout le monde part à Jakiri. Cette situation est encore favorisée par l'usage de l'anglais comme langue de communication administrative et du pidgin comme lingua franca pour une population totalement anglophone, contrairement à Bangourain qui est francophone. Dès lors, l'on assiste à une déconnexion de ces deux villages de l'ensemble de l'arrondissement de Bangourain et à une forme de solidarité avec l'arrondissement de Jakiri, duquel ils sont desservis par une route. 
Localisation du village Pouakam dans les royaumes bamoun (département du Noun) et banso (département du Bui)

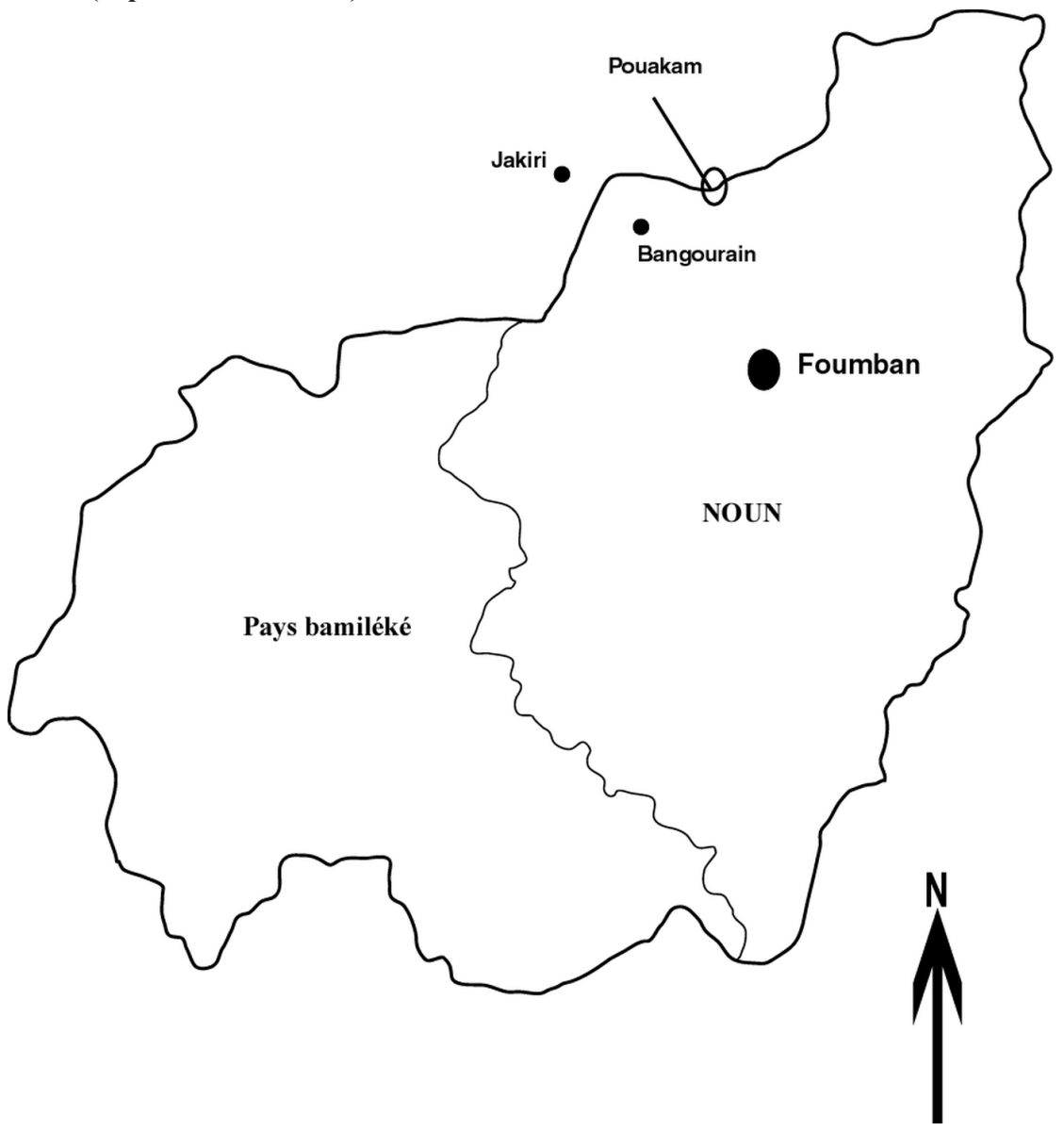

L'étude porte essentiellement sur les Pouakam du royaume bamoun et puise sa source dans les travaux de longue date que nous menons dans la région de l'Ouest sur les groupes dominants que sont les Bamoun et les Bamiléké. C'est au travers de ces travaux que récemment, nous avons opéré un revirement vers la problématique des minorités ethniques ${ }^{7}$. Cette orientation

7 Voir Mouiche, Ibrahim,-Démocratisation et intégration sociopolitique des minorités ethniques au Cameroun. Entre dogmatisme du principe majoritaire et centralité des partis politiques, 2012, Dakar; « Democratisation and Political Participation of Mbororo in Western Cameroon" in Africa Spectrum 2, 2011, pp. 71-97; « Autochtonie, libéralisation politique et construction d'une sphère publique locale au Cameroun » in African Studies Review, Volume 54, Number 3, December 2011, pp. 71-99. 
nous a exigé une certaine ouverture sur l'interaction entre Pouakam et leurs voisins et « hôtes » bamoun et banso. Dans cette perspective notre démarche s'est voulue exploratoire. Pour y parvenir, nous avons fait appel à trois techniques de recherche : les techniques documentaires, les interviews et des focus group discussions. D'abord nous avons consulté les sources documentaires constituées d'écrits portant sur les Pouakam, les Bamoun, les Banso, les Tikar. Et c'est le lieu de déplorer ici qu'avant notre ouvrage publié en 2012, il n' existait aucun écrit sur les Pouakam. Les sources primaires se résument à l'essentiel aux archives administratives et municipales consultées à Bangourain, le chef-lieu de l'arrondissement dont dépendent les Pouakam du Noun. L'enquête par interviews semi-directives et parfocus group nous a conduit à Bangourain, à Jakiri et à Koupouakam I et II. Les discussions ont été ainsi menées auprès des autorités administratives, politiques et traditionnelles et de quelques citoyens ordinaires, des natifs pouakam, bamoun et banso. Après un détour théorique qui nous permettra de mieux cerner la singularité de l'autochtonie des Pouakam, suivront successivement leur procès migratoire et les déterminants de cette autochtonie qui restent largement méconnus. Cette dernière partie sera renforcée par quelques esquisses comparatives.

\section{A. Quelques préalables théoriques sur l'autochtonie et les migrations}

L'autochtonie est devenue aujourd'hui en Afrique « une référence pour affirmer ou réaffirmer des droits exclusifs sur la terre ancestrale et bénéficier d'un accès privilégié aux ressources qu'ils offrent. Est mis en avant le droit du premier occupant » ou des conquérants régulant l'accès à la terre dans le monde rural en tant qu'autorités coutumières, selon des procédures négociées sans règles codifiées ${ }^{8}$. Elle implique donc la notion de territoire, avec les droits fonciers particuliers qui peuvent s'y rattacher. Celui qu'on appelle « autochtone » est l'occupant par voie héréditaire, le plus ancien des occupants actuels, voire le « premier», si tant est que cela soit vérifiable ${ }^{9}$. L'autochtonie se traduit simultanément par des comportements de rejet, des représentations symboliques et de la culture matérielle de l'autre. Elle se manifeste aussi « non seulement pour investir au village ou y faire valider sa réussite, mais aussi pour s'y enterrer, ce critère devenant le véritable terroir $»^{10}$. A la suite de Carola Lentz, on peut aussi voir la distinction entre hôtes et étrangers comme un mode de relation qui distingue les groupes selon un principe d'autochtonie. « La catégorie de 'l'étranger' a été, et reste aujourd'hui, définie en opposition aux deux principes d'appartenance: la communauté rituelle,

8 Médard, Claire, "Il existe un droit ancestral à la terre des communautés dites 'autochtones' » in Courade, Georges, L'Afrique des idées reçues, Paris, 2006, p. 166-167.

9 Lespinay, Charles de, «Autochtonie et droit foncier : 1'existence contestée des Banynunk en Afrique de l'Ouest » in Droit et cultures, $\mathrm{n}^{\circ} 32,1996$, p. 55.

10 Voir Bayart, Jean-François, Geschiere, Peter et Nyamnjoh, Francis. B., «Autochtonie, démocratie et citoyenneté en Afrique » in Critique internationale $n^{\circ}$ 10, p. 177-194; Geschiere, Peter, The Perils of belonging. Autochthony, Citizenship, and Exclusion in Africa and Europe, Chicago and London, 2009. 
constituée autour d'un autel de terre, et le groupe de parenté» ${ }^{11}$. Parce qu'elle un processus récent d'inclusion-exclusion à fondement spatial s'inscrivant contre la fluidité des frontières et le métissage national ${ }^{12}$, l'autochtonie est devenue un rival dangereux de la citoyenneté nationale ${ }^{13}$

L'autochtonie se décline sur des modes divers : de l'ethnicisation aux nationalismes, des rhétoriques de l'appartenance à l'ancestralité, sa force semble résider surtout dans sa capacité à articuler différents niveaux d'identité dans un contexte de volatilité des processus politiques et économiques du capitalisme contemporain. Derrière cette multiplicité, soutient Armando Cutolo, on peut voir les rapports de force et les luttes qui investissent la citoyenneté et donc l'État, dans le cadre d'une logique biopolitique du gouvernement de la vie, des «populations » et des «appartenances » avec des « territoires » et des « ressources», suscitant des réponses et des résistances qui sont profondément imbriquées ${ }^{14}$. Aussi loin d'être un archaïsme, le principe d'autochtonie et la valorisation de l'ancrage sur un territoire sont-ils devenus des éléments clefs dans l'organisation de la vie collective en milieu urbain et même des adjuvants pour accéder à un plus haut niveau d'urbanité. Pouvoir valoriser son ancrage autochtone, c'est aussi s'assurer un accès plus grand aux ressources d'une agglomération. Ces enjeux pluriels, économiques, symboliques et sociaux apparentent l'autochtonie à une forme de capital qu'il convient d'investir et de rentabiliser ${ }^{15}$. Dans ces conditions, la montée en puissance de l'autochtonie apparaît bien comme une nouvelle phase de l'ethnicité, dont les termes antagonistes gardent la même capacité émotionnelle à créer une opposition entre «nous» et " eux », mais ont l'avantage d'être moins spécifiques et donc plus faciles à manipuler que les anciens récits historiques, trop riches pour ne pas autoriser des recompositions, des déconstructions et des interprétations concurrentes de l'identité et des origines ${ }^{16}$.

S'agissant des migrations, schématiquement l'on distingue les migrations anciennes et les migrations contemporaines. Sur cette base, Jean Loup Amselle dans la théorie du phénomène migratoire africain, lie les migrations modernes à une évolution économique qui favoriserait

11 Lentz, Carola, «Premiers arrivés et nouveaux venus. Discours sur l'autochtonie dans la savane ouestafricaine » in Kuba, Richard, Lentz, Carola, Nurukyor, Claude et Somda (eds.), Histoire du peuplement et relations interethniques au Burkina Faso, Paris, 2003, p. 119.

12 Médard, note 7, p. 166-167.

13 Voir Geschiere, note 9; Geschiere, Peter, "Autochthony and the Crisis of Citizenship" in African Studies Review, vol. 49, $\mathrm{n}^{\circ}$ 2, 2006, p. 1-7; "Autochthony and Citizenship: New Modes in the Struggle over Belonging and Exclusion in Africa" in Quest, vol. 18, $\mathrm{n}^{\text {os }}$ 1/2, 2004, p. 9-23; Konings, Piet, "Religious Revival in the Roman Catholic Church and the Autochtony-Allochtony Conflict in Cameroon » in Africa 73 (1), 2003, p. 31-56; Ceuppens, Bambi et Geschiere, Peter, " Autochthony: Local or Global? New Modes in the Struggle over Citizenship and Belonging in Africa and Europe" in Annual Review of Anthropology, Vol. 34, 2005, pp. 385-407.

14 Cutolo, note 3, p. 6-7.

15 Hilgers, Mathieu, «L'autochtonie comme capital: appartenance et citoyenneté dans l'Afrique urbaine » in Social Anthropology, Volume 19, Issue 2, May 2011, p. 144.

16 Bayart, Jean-François, Geschiere, Peter et Nyamnjoh, Francis. B., "Autochtonie, démocratie et citoyenneté en Afrique » in Critique internationale n²10, janvier 2001, p. 180-181. 
surtout le déplacement de main-d'œuvre ou d'éventuels travailleurs; par contre, «les migrations anciennes ou archaïques » s'opposeraient aux « migrations modernes » en ce qu'elles représentent plutôt un mouvement d'expansion ou de déplacement d'une société donnée pour des causes qui tiennent essentiellement aux caractéristiques mêmes de cette société ${ }^{17}$. Il s'agit toujours d'un flux « collectif » mais surtout « irréversible ${ }^{18}$. Ce mouvement peut être inévitable, forcé : catastrophes naturelles, processus écologiques... ou imposé : expulsions, réfugiés..., mais aussi parfois volontaire : rupture avec le milieu social d'origine, choix d'un mode de vie professionnel, culturel social différent. Il se solde toujours par la perte du statut d' « étranger » et l'acquisition de celui d'autochtones. Raison pour laquelle, aujourd'hui, à en croire avec raison Hervé Domenach and Michel Picouet, des mouvements collectifs et irréversibles de cette ampleur ne peuvent plus avoir lieu, eu égard à la raréfaction des terres encore vierges et à la stabilité des frontières territoriales des Etats voire des communautés ethniques $^{19}$.

Nous avons vu plus haut que l'autochtonie se spécifie par le droit du «premier occupant», c'est-à-dire des groupes ou les ethnies qui étaient « déjà là » lors de contacts ultérieurs avec des « peuples migrateurs »; comment déterminer maintenant le « premier occupant »? A cette question trois thèses s'affrontent : celle du « désert humain » impliquant une migration fondatrice : selon cette thèse, « aux origines, il n'y avait rien; aucune présence humaine » puisque « au départ il n'y avait rien »; il y a ensuite la thèse migrationniste et celle de l'autochtonie « originelle » ou « endogène » ou de la continuité ethno-territoriale (continuité ethnique dans le même lieu). Bien évidemment, l'analyse des récits de fondation témoignent de l'inexistence de véritables autochtonies dans la mesure où ils font presque tous état de mythes fondateurs, donc de migrations fondatrices. Simplement, ces migrations fondatrices peuvent impliquer diverses modalités de contacts inter-ethniques, sauf le cas du désert. Mais les thèses de l'autochtonie comme les thèses migrationnistes, au-delà de leurs divergences portant sur le point de savoir qui était le premier à tel endroit, s'accordent sur l'importance à accorder à la notion de continuité, continuité prouvée et reconstruite par tous les moyens, y compris par le cumul temporel et/ou spatial de discontinuités ${ }^{20}$.

A ces trois thèses, il faut impliquer pour l'Afrique qui a connu l'invasion européenne comme dans d'autres régions semblables du monde, le moment colonial comme point d'ancrage ou d'arrimage du statut d'autochtone. Comme l'on sait, dans ce continent, la répartition actuelle des différents groupes ethniques est largement héritée des divisions spatiales antérieures à l'époque coloniale. Or, soutient B.Tallet dans son étude sur le Burkina Faso ${ }^{21}$, à la

17 Amselle, Jean-Loup, (dir), Les migrations africaines : réseaux et processus migratoires, Paris, 1976.

18 Voir Domenach, Hervé et Picouet, Michel, «Le caractère de réversibilité dans l'étude de la migration » in Population (French Edition), 42 e Année, No. 3 (May - Jun., 1987), p. 469-483.

19 Domenach et Picouet, note 17, p. 474.

20 Guillorel, Hervé et Michels, Patrick, « Continuité territoriale, continuité nationale. L'exemple yougoslave" in Balkanologie, Vol. I, n 1, juillet 1997, p. 1-25.

21 Tallet, B., «Espaces ethniques et migrations : Comment gérer le mouvement? » in Politique africaine $\mathrm{n}^{\circ} 20,1985$, p. 65. 
notion de frontière ethnique jugée « inexacte car trop précise », il faut plutôt opposer celle de « marche, séparant des ensembles ethniques ». Ces marches sont fluides, mobiles en fonction de rapports de force, souvent belliqueux : la puissance militaire, a ainsi favorisé l'expansion de certains groupes. Cette fluidité des divisions spatiales a été interrompue par la colonisation au cours de laquelle l'autochtonie devint un principe de catégorisation permettant d'identifier puis d'administrer des populations ${ }^{22}$. Par la fixation de limites territoriales, l'identification administrative et le dénombrement des populations, la distinction entre les primo-arrivants et arrivants tardifs s'est affermie et la dynamique du peuplement a été modifiée. L'articulation entre propriété du sol, autochtonie et souveraineté politique a affecté l'organisation sociale et les rapports de force. Dans certains cas, l'autochtonie a épousé les nouvelles circonscriptions administratives, dans d'autres elle a recouvert les variables ethniques co-produites par l'administration et les populations locales ${ }^{23}$. La paix coloniale ayant ainsi figé des espaces auparavant mobiles, depuis lors, l'immobilisme a succédé à l'instabilité et les limites ethniques n'ont pas évolué. Il en a résulté comme conséquence, l'exigence d'une continuité historique avec une région déterminée avant la colonisation et un lien étroit avec ses terres, pour qu'un groupe puisse bénéficier du statut d'autochtone. Ce qui explique aussi pourquoi l'une des singularités africaines des idéologies de l'autochtonie consiste en leur teneur ethnique, quand d'autres mouvements comparables, en Europe notamment, mettent en exergue les clivages culturel, linguistique, religieux, national ou régional ${ }^{24}$. Si aujourd'hui les migrations collectives surviennent ou peuvent toujours survenir, il s'agit surtout des migrations forcées toujours réversibles même illusoirement parce qu'insusceptibles de conférer aux déplacés, le statut d'autochtones. D'ailleurs, rarement ou nullement, celles-ci ne mobilisent toute une échelle ethnique ou clanique comme autrefois. Dès lors, comment comprendre l'autochtonie pouakam intervenue bien après l'indépendance du Cameroun? Que retenir de cette trajectoire singulière?

\section{B. La migration pouakam : une migration collective, ethnique, post-indépendance et irréversible}

L'orientation par une partie des recherches anthropologiques qui, en Afrique, s'étaient lancées dans la reconstitution de l'histoire des groupes doit se poursuivre et s'approfondir. Depuis de nombreuses décennies, on fait grand cas des traditions orales et on en tire un grand parti de récits riches et nombreux même s'il faut reconnaître que les traditions orales, qu'aucune donnée extérieure ne vient corroborer, nous mettent en présence de faisceaux de présomptions difficiles à critiquer. Toutefois, comme l'a si bien vu Claude Tardits, l'anthropologue engagé dans un travail de comparatiste sur des thèmes de récits traditionnels, sans souci de leur his-

22 Ceuppens, Bambi et Geschiere, Peter, “ Autochthony: Local or Global? New Modes in the Struggle over Citizenship and Belonging inAfrica and Europe" in Annual Review of Anthropology, Vol. 34 (2005), p. 387-390.

23 Hilgers, note 14, p. 145.

24 Voir Bayart, Geschiere et Nyamnjoh, note 15, p. 179-180. 
toricité, est dans une position bien meilleure que l'historien puisqu'il ne s'efforce que de les rendre intelligibles les uns par les autres et de percevoir le mouvement par lequel ils se développent, ne leur demandant jamais ce qu'ils ne contiennent pas, la garantie de leur fidélité à l'histoire. La reconstitution historique a des exigences qui ne peuvent faire fi, en Afrique, d'une quelconque possibilité de corroborer les données de l'oralité ${ }^{25}$. C'est ainsi que les témoignages que nous avons recueillis sur le terrain permettent de se représenter l'histoire des Pouakam; celle-ci s'articule autour de la question de l'ethnogenèse considérée comme l'étude dynamique des impacts des migrations, des conflits et autres processus de multiplication des « contacts » entre groupes, sur les renforcements voir même la genèse des identités communautaires ${ }^{26}$.

1. Les Bamoun et les Tikar comme composantes ethniques autochtones originelles du royaume bamoun

Si le principe d'autochtonie gagne en importance cela ne signifie pas qu'il soit aisé de déterminer qui est, ou non, autochtone. D'un point de vue de l'ethnogenèse, le royaume bamoun comprend aujourd'hui trois composantes ethniques autochtones : les Bamoun, groupe majoritaire et les minorités tikar ${ }^{27}$ et pouakam. Pourtant, à l'indépendance du Cameroun, les Pouakam ne faisaient pas encore partie de ce royaume. Ils étaient plutôt établis dans l'actuelle région anglophone du Nord-Ouest. Ce que l'on sait est qu'au XVI ${ }^{\mathrm{e}}$ siècle se produit un événement majeur, la création du royaume bamoun: à la suite d'une dispute de trône, le prince tikar Nchare Yen quitte Bankim avec une armée de partisans, conquiert ou rallie des populations trouvées sur place, et fonde un petit royaume qui a pour capitale l'actuelle ville de Foumban, s'étendant tout autour du chef-lieu dans un rayon variant de 20 à $30 \mathrm{~km}$ et occupant une surface approximative de $400 \mathrm{~km}^{2}$. Pendant environ deux cents ans, neuf souverains se

25 Tardits, Claude, «L'espace, indicateur historique, révélateur structural: l'exemple bamoum (Cameroun) » in Annales. Histoire, Sciences Sociales, 40 e Année, No. 6, 1985, p. 1262.

26 Voir Gonzalez, Nancie L. et McCommon, Carolyn S., eds., Conflict, migration, and the expression of ethnicity, Boulder, 1989; Fardon, Richard, " 'African ethnogenesis' : limits to the comparability of ethnic phenomena", in Holy, Ladislav, ed., Comparative anthropology, Oxford, 1987, p. 168-188.

27 On appelle communément plaine tikar, du nom des populations qui l'occupent, une dépression située dans la partie occidentale de la République du Cameroun. Elle s'adosse, à l'ouest et au nord respectivement, au massif du Mbam et aux premiers contreforts, assez abrupts, du plateau de l'Adamaoua; à l'est et au sud, elle est l'aboutissement d'une vaste trouée drainée par les principaux systèmes fluviaux du centre du Cameroun, cours du Djerem, de la Sanaga et de la Bénoué. Du point de vue ethnique, les limites actuelles de l'extension des Tikar coïncident avec celles des peuples suivants, pour autant que les constants déplacements permettent encore d'identifier des territoires spécifiques : à l'ouest, les Bamoun, au nord-ouest, les Mambila, au nord, les Foulbé, au sud et au sud-est, les Babouté, dont le principal centre, Yoko, est tout proche du pays tikar. Les Tikar débordent, au nord du pays bamoun, sur le Cameroun anglophone. On rencontre même quelques groupes, au-delà de la frontière, au Nigeria. Mais le territoire où leurs traditions et leur langue paraissent le mieux conservées est représentée entre Foumban et Yoko, par la bande qui jalonne, du nord au sud, les grandes chefferies de Bankim, de Ngambé et de Nditam (voir Hagege 1969 : 11). Ils sont répartis, du point de vue administratif, dans quatre régions du Cameroun: l'Adamaoua, le Centre, l'Ouest et le Nord-Ouest. 
succèdent à la tête du pays dont la situation territoriale ne change pas. Dans la première moitié $\mathrm{du} \mathrm{XIX}^{\mathrm{e}}$ siècle, les Bamoun sous le règne de Mbouombouo repoussent les Peuls venus de l'Adamaoua, guerroient et portent leurs frontières au Noun et au Mbam, absorbant un effectif de population plus élevé que le leur. Le royaume s'étendit ainsi, atteignant la taille qu'il conservera dans toute la suite de son histoire. Les Bamoun réussirent donc à constituer un royaume, mais ce passage se fit dans la violence. Or, une grande partie de la population n'accepta pas cette domination brutale et préféra émigrer. Ce qui provoqua un vide démographique encore visible aujourd'hui. Les chefs conquis le furent dans une telle situation d'inégalité qu'ils perdirent la plupart de leurs fonctions et ne purent maintenir un minimum d'autonomie. Ils furent ravalés au rang de simples notables ${ }^{28}$.

Ce système manifeste une grande originalité par rapport à ses homologues bamiléké et bamenda dominés par les chefferies ${ }^{29}$. Les chefs conquérants bamoun ont su acquérir et utiliser un pouvoir mystique et, à la faveur de ce pouvoir, ils ont pu appliquer une habile politique d'unification. Le problème était double: il fallait assimiler les peuples conquis, mais en même temps organiser une vie politique suffisamment active et centralisée pour éviter que des morcellements ou des sécessions ne remettent en question à chaque génération les résultats acquis. Mais il ne suffisait pas de conquérir une vaste région et d'en faire un tout cohérent. Il fallait encore lui donner une organisation intérieure assez solide pour résister aux tendances décentralisatrices. La recherche d'un pouvoir absolu et centralisé semble avoir été une préoccupation des rois ${ }^{30}$. Devant leur politique d'unification, les souverains bamoun n'ont pas cru combattre ou assimiler les Tikar, pour des raisons liées à leurs origines communes sûrement. S'il y eut eu antagonisme entre les deux groupes, les Tikar auraient soit émigré, soit se seraient soumis, abandonnant leur parler originel comme tous les autres communautés, et ne constitueraient pas aujourd'hui une minorité ethnique du département du Noun, mais des Bamoun tout court. Mais malgré cette cohabitation en bonne intelligence entre la royauté bamoun et les Tikar, la prétention hégémonique d'un des chefs tikar donna l'occasion au souverain bamoun d'étendre sa suzeraineté sur les groupes tikar environnants comme le souligne Claude Tardits :

"Les peuples installés au nord-est du pays, le long du Mbam et surtout de la Mapé eurent un sort différent de la quasi-totalité des précédents. Les principicules fixés dans cette région furent en effet soumis sans combat dans la plupart des cas; aussi les ha-

28 Tardits, Claude, « Parenté et pouvoir politique chez les Bamoun (Cameroun)» in L'Homme, Revue française d'anthropologie, janv-juin 1973, p.39; « Le royaume bamoun: un Etat africain traditionnel qui a traversé toute l'histoire » in Mondes et cultures, t 52, no 2-4, 1992, p. 674; voir Dubié, Paul, "Christianisme, islam et animisme chez les Bamoun (Cameroun)» in Bulletin de l'IFAN, Série B, Sciences humaines, Dakar, tome XIX, juil-oct 1957, p.338; Champaud, Jacques, « Genèse et typologie des villes du Cameroun de l'Ouest » in Cahiers ORSTOM, série sciences humaines, vol IX, no 3,1972, p. 327.

29 Warnier, Jean-Pierre, L'esprit d'entreprise au Cameroun, Paris, 1993, p. 202-203.

30 Binet, Jacques, « Le commandement chez les Bamoun » in Bulletin de l'IFAN, Série B, Sciences humaines, Dakar, IFAN, tome XIX, juil-oct 1957, p. 400-409. 
bitants restèrent-ils sur place.... Le plus puissant de tous ces anciens émigrés était, à l'époque, le chef nte qui, se disant issu du lignage royal ... de Mbankim, s'efforçait de reconnaître sa souveraineté par les voisins dont il prétendait recevoir un tribut. Cette tentative d'hégémonie régionale aurait favorisé l'expansion bamoun, car plusieurs chefs sollicitèrent l'intervention de Mbouombouo pour échapper aux exigences du souverain nte; le roi bamoun réduisit cet ambitieux .... L'ensemble de ces roitelets tombèrent alors sous la domination des Bamoun, tout en conservant un statut des 'rois soumis' (nfontue) qui les laissait en place $»^{31}$.

Ce que Claude Tardits ne mentionne pas, c'est que contrairement à la politique d'unification imposée aux autres groupes conquis ou soumis, les Tikar du royaume bamoun conservèrent leurs langues. La paix coloniale qui va fixer et délimiter les frontières des groupes ethniques va avaliser ces arrangements politico-institutionnels et depuis lors, ceux-ci sont devenus une minorité autochtone du royaume bamoun même si pendant longtemps, de 1960 à 1981, c'est le terme « département bamoun » qui désignait cette aire qui épouse comme nous l'avons souligné plus haut les contours du royaume bamoun. C'est le décret $\mathrm{n}^{\circ} 81 / 522$ du 11 décembre 1981 qui impose une nouvelle appellation, « département du Noun », du nom du fleuve Noun, entourant dorénavant cette dénomination administrative dorénavant d'une aura d'impartialité neutralisant les particularismes locaux, tant, en dehors des Bamoun, l'on y retrouve d'autres groupes autochtones: les Tikar et depuis les années 1960, les Pouakam.

\section{De l'ethnogenèse des Pouakam}

L'histoire des Pouakam est une longue pérégrination à inscrire dans les migrations forcées imposées par le souverain bamoun Mbououmbouo. Remettons-nous une fois à Claude Tardits qui évoque la fuite de ce groupe (appelés ici Pa Zrum- équivalent à Nzerem vu plus haut) :

« Les populations établies à l'ouest et au nord du massif du Nkogham puis au pied de celui du Mbam furent également rejetées au-delà du Noun: les Pa Nden, les Pa Ghaam, les Pa Pa avec lesquels Nchare avait déjà eu maille à partir, les Pa Mbowon, les $\underline{P a}$

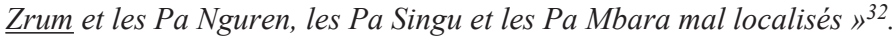

Selon les informations recueillies sur place, au départ semble-t-il, le groupe originel constitué des Nchingong vivait toujours dans l'actuel site de Pouakam sur les piedmonts du massif Mbam bien avant la fondation du royaume bamoun. Mais les guerres de conquête de Mbouombouo vont contraindre les Nchingong à émigrer outre-Noun, d'abord vers Jakiri et même à Kumbo (actuel pays banso) puis à Bapessi dans la plaine de Ndop (actuel département de Ngo Ketunja). On retrouve encore aujourd'hui un fond pouakam resté à Kwanso dans la frontière entre le département du Bui et celui de Ngo Ketunja. A l'époque qui nous concerne, le groupe Nzerem n'existait pas; les Pouakam constituaient une et une seule communauté: les Nchin-

31 Tardits, Claude, Le Royaume bamoun, Paris, 1980, p. 142 et 144.

32 Tardits, note 30, p. 142. 
gong. C'est sous la colonisation allemande (1884-1916) qu'intervint la scission. Ce fut à la suite d'une dispute entre le chef nchingong et son petit frère, où ce dernier quitta Nchingong, groupa des partisans et fonda le groupe nzerem. Nchingong garde donc le parler originel et aujourd'hui, ces relations parentocratiques renforcent la vie en bonne intelligence entre les deux communautés.

La migration vers l'actuel site de Pouakam et la fixation définitive, véritable retour au bercail, pour ainsi dire, est toutefois récente, datant de 1968. Si par l'émigration, les Nchingong avaient pu échapper à leur soumission et conquête par les Bamoun, ceux-ci demeuraient à l'étroit à Bapessi et toujours considérés comme des " allogènes » voire des « étrangers ». Devant cet environnement hostile, ils décidèrent de se trouver un espace propre, habitable, arable et suffisamment vaste pour accueillir tout le monde. L'on se souvint alors que depuis l'émigration du XIX ${ }^{\mathrm{e}}$ siècle, Koupouakam demeurait un véritable no man's land puisque, aucune communauté ethnique, pas même les Bamoun, et a fortiori les Banso, n'était venue occuper cette cuvette. C'est alors que sous la sollicitude du sultan-roi des Bamoun, Njimoluh Seidou, les Nchingong partent de Bapessi avec leur chef en la personne de Nguenkam Yacouba, traversent le territoire banso et se réinstallent dans leur site de Koupouakam. Quelque temps après, ils sont rejoints par le chef Nzerem, parti de Banso avec son peuple.

Une fois réinstallés, le chef supérieur du groupement Njinka dont dépendent de nombreux villages de l'arrondissement de Bangourain et le chef de Bangourain, village attenant quelque peu Koupouakam, avaient voulu établir sur les Pouakam leur suzeraineté. « Pour nous libérer de cette tutelle et surtout nous autoriser légalement à nous installer sur ces terres, et surtout y disposer de la pleine jouissance et propriété de la tenure foncière, le sultan nous délivra cette attestation » soutient le chef des Nchingong, sa Majesté Fon Fobah Elias de Koupouakam I lors de notre entretien. Voici les termes de ladite attestation qui date du 24 septembre 1971 :

« Je soussigné El Hadj Seidou Njimoluh Njoya, Sultan des Bamoun, Maître traditionnel des terres bamoun et Maire de Foumban,

«Certifie par la présente que la portion de terrain à Pouakam 1 (Foumban) appartient à Monsieur Nguenkam Yacouba, titulaire de la carte d'identité no 004/1108/69 délivrée par Ndzomo Victor, Commissaire spécial.

" Le nommé Nguenkam Yacouba est seul autorisé à s'y installer sur ce terrain à Pouakam et ce terrain devient sa propriété avec les descendants de sa famille.

«En foi de quoi, la présente attestation est délivrée pour servir et valoir ce que de droit».

Malgré les précautions d'usage dans la plume du sultan, cette migration et installation qui ne concerne qu'une famille, va se muer en une fixation définitive d'un groupe ethnique : les Pouakam. Par cette migration irréversible, ceux-ci vont acquérir le statut d' " autochtones » du royaume bamoun, à l'instar des Bamoun et de la minorité tikar. Cette étude de l'interaction 
entre la royauté bamoun et les Pouakam nous donne une autre dimension au débat sur l'ethnicité et permet aussi d'élargir la réflexion sur la notion d'autochtonie.

\section{Les déterminants de l'autochtonie des Pouakam}

La migration récente des Pouakam s'étant soldée par l'acquisition du statut d'autochtones, il ne fait aucun doute, cette migration s'inscrit plutôt dans le cadre des « migrations anciennes ou archaïques ». Quels sont les déterminants de cette autochtonie? Bien plus, l'autochtonie étant devenue avec la libéralisation politique une forme de capital qu'on peut investir et rentabiliser, quelles en sont les conséquences juridiques pour la représentation politique des Pouakam?

\section{Du rôle central de la royauté bamoun dans la déterminantion de l'autochtonie pouakam}

Une question nous taraude l'esprit à ce niveau: les autochtones étant ceux qui étaient présents « avant »..., continuité assumée par le moment colonial, quels sont les déterminants de l'autochtonie des Pouakam? Qu'est-ce qui entre en ligne de compte comme critères? La race, l'antériorité dans un territoire donné, le fond culturel, les mythes précoloniaux de fondation, etc., dans une approche primordialiste de l'ethnicité? D'une part, avant sa domestication par les immigrants pouakam, cette localité était un no man's land, toutes choses qui semblent conforter l'hypothèse du « désert humain » impliquant une migration fondatrice. Comme le souligne Mathieu Hilgers, le principe d'autochtonie ne peut nullement être réduit à son caractère strictement démographique: être autochtone ce n'est pas seulement et, dans certains cas, ce n'est pas du tout, être premier dans l'ordre des migrations. Si le principe d'autochtonie renvoie toujours, au moins formellement à cet ordre, c'est parce qu'il suppose que les premières populations arrivées sur un espace sauvage l'ont rendu humainement viable en combattant, en domestiquant ou en s'alliant les forces naturelles visibles et invisibles qui y régnaient. Derrière la question démographique, le principe d'autochtonie renvoie à une dimension sociologique, à savoir: la contribution d'un groupe à la prospérité d'une collectivité résidant sur un espace circonscrit. Le premier groupe à être arrivé sur un espace est bien le premier à l'avoir civilisé ${ }^{33}$. Seulement, selon les Bamoun, depuis les conquêtes du roi Mboumbouo du XIXe siècle, cet espace fait partie intégrante du royaume bamoun et appartient à son peuple même s'il n'était pas habité. No man's land ne signifie nullement terra nullius (terre sans maître), ce qui explique d'ailleurs pourquoi l'installation des Pouakam ne fut possible que par l'accord exprès du sultan-roi des Bamoun, à la demande de ceux-ci. Néanmoins, il convient de le reconnaître, ce contexte de désert humain a facilité cette fixation collective et surtout servi de prétexte pour acquérir le statut d'autochtones. Il est certain que si cet espace était habité et domestiqué par les Bamoun, il eut été difficile voire même impossible pour les Pouakam d'acquérir ce statut quand même ils se seraient établis collectivement ou ethnique- 
ment. Ils seraient aujourd'hui plutôt rangés dans la catégorie des allogènes du royaume bamoun, à l'instar d'autres groupes immigrants comme les Bamiléké ou les Banso.

D'autre part, il y a l'hypothèse de la fixation antérieure, précoloniale et préalable des Pouakam dans ce lieu, et donc des « descendants des premiers occupants »; mais à l'image du premier argument, outre que cette évocation est discutable, celle-ci n'est pas contraignante pour le roi bamoun, descendant des « conquérants ", régulant l'accès à la terre du royaume en tant qu'autorité coutumière disposant de la souveraineté politique traditionnelle. Les Pouakam n'ont-ils pas perdu l'initiative depuis les conquêtes de Mbouombouo, quand en fuite, ils ont abandonné ce terroir? On voit toutefois ici dans la perspective migrationniste l'hypothèse de la continuité mobile avec leur retour dans cette localité, aussi vérifiable que puisse être cette ethnogenèse. Mais une fois de plus, ce retour n'a été possible que par l'assentiment du sultan-roi des Bamoun.

Quid enfin du fonds commun culturel tikar ${ }^{34}$, et en voie de conséquence, la race semiebantoue, bantoïde? Cette proximité culturelle voire parentocratique avec les Bamoun est attestée par le parler pouakam très voisin de la langue bamoun. Cependant, si l'on s'en tient à ces variables, de nombreux Bamiléké 35 résidant aujourd'hui en pays bamoun ou les Banso du village Koutoukpi dans l'arrondissement de Bangourain seraient des autochtones du royaume bamoun, ce qui n'est et n'a jamais été le cas; c'est pour cette raison qu'en dehors de cette approche primordialiste de l'ethnicité, il faut faire valoir la perspective subjectiviste ou in-

34 D'un point de l'ethnogenèse et des mythes de fondation, les Tikar auraient essaimé de nombreux groupes ethniques, lesquels se réclament toujours cette origine. Raison pour laquelle se dire d'origine « tikar » peut simplement signifier qu'on est originaire du pays aujourd'hui nommé tikar mais peut aussi indiquer une parenté avec le lignage royal qui fut à l'origine de la naissance de ce peuple. C'est surtout la littérature de langue anglaise qui nourrit la controverse en entretenant l'amalgame entre l'ethnie tikar proprement dite qui a pris cet ethnonyme, et les communautés ethniques apparentées, disséminées dans la zone de langue anglaise (the tikar tribes) : Ndop, Mbem-Nsungli, Bum, Bafut, Kom, Fungom et Nso. Cet amalgame est tout simplement une aberration; l'ethnonyme tikar individualise un groupe qui se considère comme tel, parle une même langue bien que réparti dans quatre régions du Cameroun. Si l'on devrait d'ailleurs souscrire à une telle généralisation, elle resterait superflue de ne pas intégrer, côté francophone, les Bamoun, autre groupe d'origine tikar, constitué à la même période migratoire et sous la poussée des mêmes événements que les Nso. Le terme Ndop quant à lui, n'est à proprement parler pas un ethnonyme, mais une localité regroupant plusieurs communautés d'origine tikar aux parlers distincts.

35 En vue de résorber la forte concentration démographique des Bamiléké sur les hauts plateaux de l'Ouest, des implantations permanentes de ceux-ci furent organisées dans le royaume bamoun dans les années 1920-30 par l'administration française et des villages de colonisation furent ainsi créés dans la rive gauche du Noun portant le nom des chefferies bamiléké d'où venaient ses habitants: Bangou II, Bamendjou II, Batoufam II, etc. A ceci, il faut ajouter une colonisation qui fut plus diffuse et moins dirigée par l'administration dans l'actuel arrondissement de Kouoptamo parmi les espaces laissés vacants par les plantations européennes. Reste aussi à signaler la colonisation agricole de la région de Magba où les Bamiléké peuplent plusieurs villages de la plaine tikar: Magba et Ngounso, colonisation qui le fut dans les années 1950 avec la bénédiction d'un chef supérieur, membre de la famille royale (Nji Adamou Pokassab (adjoint au sultan-roi des Bamoun à l'époque et chef supérieur de Magba). Selon Jacques Champaud, celle-ci visait à grossir la population dont Pokassa avait reçu la charge et développer son canton. Champaud, note 4, p. 134-135. 
teractionniste. Cette approche insiste sur l'importance des interactions sociales dans la définition de l'identité car c'est en ayant des rapports avec un « autre » qu'on prend conscience de sa propre différence, et qu'on développe un sentiment nous-eux (us and them). Les groupes ethniques les mieux définis ne sont donc pas les plus isolés (point de vue primordialiste) mais au contraire ceux dont les membres ont le plus de contacts avec d'autres groupes ${ }^{36}$. En clair, dans leur interaction avec les Bamoun, les Pouakam en sollicitant leur installation en pays bamoun, se projetaient une installation définitive et surtout porteuse de l'identité autochtone, le tout accepté, avalisé et intériorisé tacitement par le roi bamoun représentant son peuple. En raison de son caractère relationnel, le principe d'autochtonie ne fixe pas de manière définitive une identité au groupe ou à un individu. Il reflète plutôt les rapports des groupes entre eux. Ces rapports sont mouvants et de nombreux auteurs ont souligné «l'instabilité de ce discours qui célèbre la stabilité» ${ }^{37}$. L'audience de la problématique de l'autochtonie procède ainsi de son ambivalence, de cette capacité à adapter à une très grande diversité de situations politiques son alternative simpliste entre «nous » et « eux» dans les termes d'un jeu à somme nulle entre $l^{\prime}$ « avant » et l'« après ${ }^{38}$.

Cette dimension interantionaliste permet de comprendre pourquoi, malgré leur migration bien antérieure à celle des Pouakam, de nombreux colons bamiléké et bien d'autres groupes immigrants établis dans le royaume bamoun sont toujours considérés ici comme des allogènes $^{39}$. Plus grave est la situation des pasteurs mbororos : arrivés et établis dans les années 1910-1920 sur les hauts plateaux de l'Ouest et du Nord-Ouest en général, ceux-ci se voient toujours considérés comme des « étrangers en défaut de territorialité» ${ }^{40}$. On retrouve ici un phénomène qu'on peut observer ailleurs en Afrique de l'Ouest : il s'agit d'une sorte de seg-

36 Gausset, Quentin, Les avatars de l'identité chez les Wawa et les Kwanja du Cameroun, Thèse de doctorat en sciences sociales, Université Libre de Bruxelles, vol 1, 1997, p. 339.

37 Ceuppens et Geschiere, note 12, p. 402.

38 Bayart, Jean-François et Geschiere, Peter (sous la responsabilité de), "'J'étais là avant'. Problématiques politiques de l'autochtonie » in Critique internationale no 10, janvier 2001, p. 127; voir Geschiere, note 9.

39 On l'a vu de manière dramatique dans l'ex-Zaïre (actuelle RDC) avec les Kasaïens « refoulés » du Shaba dont se fait l'écho Roland Pourtier. Entre novembre 1992 et 1994, avec un pic en 1993, pas moins de 600000 à 800000 Congolais originaires du Kasaï furent contraints de quitter la province minière de la RDC. Cette épuration aurait causé entre 50000 et 100000 morts selon Médecins sans frontières. La plupart de ces Kasaïens victimes de ce pogrom n'eurent d'autre choix que de retourner dans la région qu'eux-mêmes ou leurs aïeux avaient quittée, souvent depuis plusieurs décennies. Réfugiées à l'intérieur de leur propre pays, ces personnes déplacées du fait de violences politicoethniques ne purent bénéficier du statut de « réfugié » tel que l'entend le HCR. Le qualificatif de « refoulé ») leur fut appliqué. Pourtier, Roland, «Les refoulés du Zaïre : identité, autochtonie et enjeux politiques » in Autrepart no 5, 1998, p.137.

40 Il faut dire en effet, que l'opposition entre les Mbororo et les agriculteurs « autochtones » (Bamoun, Bamiléké, Banso, Tikar, Pouakam) est pratiquement totale: elle est d'ordre vestimentaire, linguistique, économique et de civilisation. Les agriculteurs participent à la culture «bantoue», entendue au sens large, tandis que les Mbororo se rattachent au monde peul dispersé à travers l'Afrique occidentale sèche. Voir Mouiche, note 6, p. 71-97. 
mentation et de «graduation» de la citoyenneté dans une pluralité de statuts hiérarchisés, ethnicisés et inégaux, comme par exemple celui qui a été donné aux « Dioula » en Côte d'Ivoire ou encore la citoyenneté imparfaite des populations du Nord du Ghana, comme les Konkomba et autres, considérées comme «acéphales " ${ }^{41}$. Maintenant que les Pouakam sont des autochtones du royaume bamoun, à l'instar des Bamoun et des Tikar, quelles en sont les conséquences juridiques pour leur position, notamment avec ce renouveau constitutionnel impulsé au Cameroun depuis les années 1990, ouvertement réceptif à la problématique de l'autochtonie?

\section{Des implications juridiques certaines pour la position des Pouakam}

Sous les différentes déclinaisons de l'autochtonie se profilent les rapports de force et les luttes qui investissent la citoyenneté et donc l'Etat. Etre autochtone permet d'avoir accès à un réseau de relations relativement durables et de disposer d'une légitimité. La reconnaissance d'une identité autochtone donne un statut et un accès à des réseaux. De nombreux travaux sur le foncier indiquent que souvent, lors des opérations d'aménagement urbain, les immigrés sont défavorisés par les commissions censées assurer l'équité dans le traitement des populations. La création d'une identité sociale autour de l'autochtonie s'expérimente comme une ressource qui permet de discriminer des concurrents dans leur accès aux ressources ${ }^{42}$. Mais surtout, c'est la libéralisation du vote avec la réhabilitation des élections comme procédure réelle de dévolution d'un pouvoir effectif qui a nourri le mythe de l'autochtonie en rendant cruciales des questions comme 'Qui peut voter où?', et plus encore 'Qui peut se porter candidat? Et où? C'est bien cette articulation entre la démocratisation et les mobilisations auxquelles elle donne lieu, d'une part, et, de l'autre, la problématique de l'autochtonie qui confère à la fois à l'idée démocratique et à la restauration autoritaire leur légitimité. Ainsi, la stigmatisation de l'allogène a été, et demeure, le grand instrument de reconquête de l'opinion par les détenteurs du pouvoir autoritaire. Bien sûr, il apporte aux 'autochtones' des gains immédiats d'ordre politique, économique ou symbolique ${ }^{43}$.

Plus spécifiquement, au Cameroun, pays de diversité par excellence, la libéralisation politique des années 1990 s'est singularisée par une réforme constitutionnelle en date du 18 janvier 1996 qui institue un Etat unitaire décentralisé, reconnaît les droits des autochtones et protège les minorités. Cette garantie des droits des minorités et des populations autochtones figure explicitement dans le préambule de la constitution. En plus, dans le titre X consacré aux collectivités territoriales décentralisées, l'article 57 (2) dispose en effet que le Conseil régional qui est l'organe délibérant de la région « doit refléter les différentes composantes sociologiques de la région ». Avant cette réforme constitutionnelle, les lois portant organisa-

41 Cutolo, note 3, p. 16.

42 Hilgers, note 14.

43 Bayart, Geschiere et Nyamnjoh, " Autochtonie, démocratie et citoyenneté en Afrique » in Critique internationale $n^{\circ} 10$, janvier 2001, pp.182-186. 
tion des élections municipales et législatives votées dans les années 1990 avaient déjà institué cette exigence de respect des « différentes composantes sociologiques » dans les circonscriptions électorales: l'article 5 (4) de la loi no 91/020 du 16 décembre 1991 fixant les conditions d'élection des députés à l'Assemblée nationale et l'article 3 (2) de la loi no 92/002 du 14 août 1992 fixant les conditions d'élections des conseillers municipaux. Idem pour le nouveau Code électoral adopté par la loi no 2012/001 du 19 avril 2012. Cette notion de « composantes sociologiques » se ramène essentiellement aux «différentes composantes ethniques de la population ». C'est d'ailleurs le sens que lui attribuent les justiciables camerounais dans le cadre des recours contentieux relatifs aux élections municipales depuis $1996^{44}$. Mais la disposition la plus révélatrice de l'ancrage au localisme du droit camerounais des minorités et des populations autochtones se situe sans doute à l'article 57 (3) qui confère aux autochtones, le droit exclusif de direction de l'exécutif régional :

«Le Conseil régional est présidé par une personnalité autochtone de la région élue en son sein pour la durée du mandat du Conseil.

" Le Président du Conseil régional est l'Exécutif de la Région ».

L'autochtonie au Cameroun semble ainsi se définir uniquement par rapport à la situation géographique des citoyens camerounais, en relation avec le découpage régional du pays : est autochtone d'une région le citoyen camerounais éligible à la présidence du conseil régional sur la base du critère de ses origines. A contrario, ne sont pas autochtones de la même région les citoyens camerounais qui ne peuvent être éligibles sur la base du critère de l'origine. Ils sont en revanche autochtones des régions dans lesquelles ils sont censés être respectivement originaires et peuvent donc y être éligibles à la présidence du conseil régional ${ }^{45}$. C'est donc dire que de par leur position d'autochones du royaume bamoun, les Pouakam en tirent de nombreuses dividendes, naturellement au plan foncier et ensuite en matière de représentation politique. Et sur ce dernier plan malheureusement, les Pouakam connaissent une très forte marginalisation. Cette communauté se singularise ici par sa position quelque peu inédite; il est ainsi curieux et triste de faire ce constat que depuis 53 ans d'indépendance, aucun Pouakam ne fait partie des effectifs de la fonction publique camerounaise prise au sens large : ni fonctionnaire, ni agent de l'Etat ou communal encore moins un entrepreneur économique, très peu de ses ressortissants ayant fait des études secondaires, conséquence de l'enclavement de leur terroir. C'est donc un peuple sans classe politique et donc sans élites, ni urbaines ni de terroir, en dehors de leurs chefs traditionnels qui sont d'ailleurs des braves paysans. Jusqu'aujourd'hui, les Pouakam sont sans représentation au sein du conseil municipal de Bangourain malgré les prescriptions législatives et constitutionnelles de respect de minorités et de composantes sociologiques de circonscriptions électorales et une commune ayant 25 sièges de

44 Donfack Sonkeng, Léopold, Le droit des minorités et des peuples autochtones, Thèse non publiée pour le doctorat en Droit, Université de Nantes, 2001, p.231.

45 Ibid, p. 306. 
conseillers municipaux ${ }^{46}$. Cette exclusion des Pouakam pose ainsi le problème de l'indispensable participation du citoyen à la légitimité du pouvoir politique, c'est-à-dire, du fossé qui sépare cette communauté et ceux qui sont chargés de conduire son destin. La représentation politique en tant que composante centrale de la gouvernance démocratique reste ainsi un défi majeur au processus de développement démocratique ${ }^{47}$.

\section{Conclusion}

Dans la théorie du phénomène migratoire africain, nous avons vu que seules les migrations anciennes étaient constitutives de l'autochtonie, elle-même porteuse de charge ethnique procédant du temps colonial. Au contraire, les migrations contemporaines s'inscrivent plutôt dans le cadre des migrations individuelles de travail; et même si des migrations collectives surviennent de temps en temps, elles seraient toujours irréversibles ou insusceptibles de conférer aux groupes immigrants le statut d'autochtones. La migration pouakam bien qu'elle soit postérieure à l'indépendance du Cameroun est plutôt à s'inscrire dans la catégorie des «migrations anciennes ou archaïques » puisque, acquisitive de l'autochtonie. Néanmoins, un décryptage approfondi du procès migratoire des Pouakam laisse apparaître des liens de filiation historique avec ce territoire où ils sont aujourd'hui installés. Ici, les thèses de l'autochtonie comme celles migrationnistes, au-delà de leur divergence portant sur le point de savoir « qui était le premier à tel endroit ", s'accordent sur l'importance à donner à la notion de continuité, continuité prouvée et reconstruite par tous les moyens, y compris par le cumul temporel et/ou spatial de discontinuités ${ }^{48}$. Les processus d'édification de l'autochtonie comme celle étatique et surtout nationale («state and nation-building ») incluent systématiquement, au-delà de leurs individuations temporelles et spatiales, un bricolage idéologique dont la fonction première est d'affirmer la continuité d'une " communauté » nationale préexistante, incréée, " déjà-la », d'où une vision atemporelle et linéaire de l'histoire prouvant, au-delà des ruptures et des discontinuités, la permanence d'une continuité qui peut être historique, linguistique, territoriale, ethnique, juridique, archéologique, etc. Nous avons ici un cas classique de « réification » ${ }^{49}$. Cette autochtonie pouakam marque ainsi un nouveau développement dans les théories migrationnistes et de l'autochtonie. Si elle s'appuie sur la thèse de « continuité mobile » et du « désert humain », elle s'en démarque par le poids qu'elle accorde à l'interaction des groupes ethniques au contraire de la thèse classique largement assise sur le déterminisme colonial. Reste à explorer la position des Pouakam du royaume banso, surtout que ceux-ci subissent les contrecoups d'une colonisation agricole récente des Banso, au contraire de ceux du royaume bamoun quasiment enfermés dans une enclave monoethnique.

46 Sur cette exclusion, lire Mouiche, Ibrahim, note 7, chap.5.

47 Omano Edigheji, "Political Representation in Africa: Towards a Conceptual Framework" in Africa Development, 2006, Vol. XXXI, No. 3, pp. 93-119.

48 Guillorel et Michels, note 19, p. 8.

49 Guillorel et Michels, note 19, p. 1. 